\title{
Biomolecular Factors Represented by Bcl-2, p53, and Tumor-Infiltrating Lymphocytes Predict Response for Adjuvant Anthracycline Chemotherapy in Patients with Early Triple-Negative Breast Cancer
}

This article was published in the following Dove Press journal:

Cancer Management and Research

\author{
Xenia Elena Bacinschi (iD) 1,2 \\ Anca Zgura (iD ${ }^{2}$ \\ Inga Safta iD ${ }^{3}$ \\ Rodica Anghel ${ }^{1,2}$ \\ 'Department of Oncology-Radiotherapy, \\ Institute of Oncology Prof. Dr. Alexandru \\ Trestioreanu, Bucharest, Romania; \\ ${ }^{2}$ University of Medicine and Pharmacy \\ Carol Davila, Bucharest, Romania; \\ ${ }^{3}$ Department of Medical Oncology, \\ Antoine Lacassagne Cancer Center, Nice, \\ France
}

\begin{abstract}
Treatment of triple-negative breast cancer is challenging. Standard adjuvant tretment is considered to be the cobination of anthracycline and taxanes although the role of anthracyclines administered preoperatively remains controversial. Actually, some studies recommended taxane-only regimens. We reviewed literatures to examine whether tissue biomarkers available in an ordinary laboratory setting (eg, haematoxylin and eosin and immunohistochemistry) may predict response to adjuvant anthracyclines in patients with triple-negative breast cancer. Our review showed that Bcl-2, p53, and tumor-infiltrating lymphocytes (TILs) expression may become independent predictors for triple-negative breast cancer. This finding was based on data from retrospective studies, and, thus, randomized controlled study is needed to confirm the present results.
\end{abstract}

Keywords: anthracyclines, biological tumour markers, triple-negative breast cancer, predictive, survival, p53, Bcl-2, TILs

\section{Introduction}

Approximately $10-20 \%$ of breast cancer is represented by the triple-negative phenotype, defined by a lack of oestrogen receptors (ERs) and progesterone receptors as well as low human epidermal growth factor receptor 2 (HER2) expression. ${ }^{1}$ Triple-negative breast cancer (TNBC), commonly diagnosed in young women ( $<40$ years), has a tendency for early relapse, with a reported five-year disease-free survival (DFS) rate of $76 \%$, even for early-stage diagnoses. ${ }^{2}$ Anthracycline-based chemotherapy regimens remain the mainstay of adjuvant treatment in this population. The 2 most commonly used anthracyclines are doxorubicin and epirubicin. Although taxanes, represented by docetaxel or paclitaxel, which have arrived historically after anthracyclines, seem to offer higher tumour shrinkage, they are still used in combination with anthracyclines. ${ }^{3}$ As several regimens are indicated in frail or older patients comprising only taxanes, the indispensability of anthracyclines in this high-risk category of breast cancer patients demands attention. Considering the long-term hematologic and cardiac toxicity induced by anthracyclines, the importance of regimen and dose density chosen in operable TNBC patients and their necessity in the adjuvant setting might be indicated by several tissue biomarkers assessable through ordinary immunohistochemistry (IHC) techniques, allowing the personalisation of treatment. ${ }^{4-6}$ However, only
Correspondence: Anca Zgura University of Medicine and Pharmacy Carol Davila, 8 Eroii Sanitari Blvd., Bucharest 050474, Romania Email medicanca@gmail.com 
topoisomerase 2-alpha has been proposed as a predictive biomarker of anthracycline treatment in HER2-amplified breast cancer. $^{7}$

In this study, we adopted an immunohistochemical method given its greater detection ability than RNA analysis in clinical practice. ${ }^{8}$ To identify the potential predictive biomolecular factors related to adjuvant anthracyclines in TNBC patients, a systematic literature review was conducted.

\section{Methods}

In the systematic review, we included retrospective and retrospective-prospective studies published as original articles, excluding case reports, case series, general reviews and editors' letters. Participants comprised adult women with unilateral, early clinical stage cT1-2N0-1/pT1-2N0-1 nonmetastatic breast cancer, either specifically triple-negative or with subgroup analysis or stratification for triplenegative, ER-negative or ER-negative/HER2-negative. Patients underwent primary curative surgery.

Studies of neoadjuvant therapy were excluded and mixed studies were included if the results were reported separately for an adjuvant setting. A histological and immunohistochemically assessment of surgical specimen for ERs, progesterone receptors, HER2 expression and adjuvant anthracycline-based chemotherapy was required. When chemotherapy protocols were mixed, articles were analysed to compare anthracycline-based regimens with non-anthracycline treatment.

Biomarkers needed to have been assessed by IHC and haematoxylin and eosin (H\&E) coloration for tumourinfiltrating lymphocytes (TILs). The number of TILs (tumor-infiltrating lymphocytes) was estimated by analyzing the mononuclear inflammatory infiltrate in the stromal regions of the analyzed tumors using $20 \times-40 \times$ targets. The infiltrate level was calculated by relating the area occupied by TILs to the total area of the tumor stroma and the results were divided into three categories: reduced inflammatory infiltrate $(0-10 \%$ of stroma), moderate inflammatory infiltrate $(10-40 \%$ of stroma) and rich inflammatory infiltrate (40-90\% of stroma).

The outcome of interest was survival (general or disease-free); thus, several variables were accepted: overall survival (OS), relapse-free survival (RFS), DFS and breast cancer-specific survival (BCSS). Overall survival (OS) was defined as the period from the date of TNBC diagnosis to the time of death with any cause. Diseasefree survival (DFS) was defined as the period from the start of treatment to the first recurrence, or to death without any type of relapse. Relapse-free survival (RFS) was defined as time from diagnosis to first relapse, local recurrence, regional recurrence, or distant metastasis. Breast cancer-specific survival (BCSS) was measured from the date of definitive breast cancer surgery to the date of death from breast cancer. To be reported in the results, at least three publications per biomarker were requested. Finally, ERs and progesterone receptors as well as HER2 expression were generally considered if assessed according to the American Society of Clinical Oncology/College of American Pathologists guidelines at the time. ${ }^{9,10}$

The search strategy consisted of checking the PubMed/ MEDLINE database and Google Scholar for original articles that met our selection criteria published since 2005 . Studies were eligible if they were written in English and reported the relationship between the biomarker and survival outcome in the adjuvant setting (DFS, RFS, BCSS or OS). A supplementary search was performed by checking the reference lists of the relevant publications. Examples of combinations of MESH terms used to define the population were "triple-negative breast cancers", "triplenegative breast neoplasm" and "ER-negative/progesterone receptor-negative/HER2-negative breast neoplasms". Additional terms employed included "limited disease", "primary operable", "T1-2N0-1 hormone-negative breast cancer", "basal-like breast cancer" and "ER-negative breast cancer". The terms used to define the intervention were "biological tumor markers", "tumor markers" and "biologic" as MESH terms and "biomolecular features" and "tumor tissue biomarker" as free expression. To define the outcome, "survival" and "predictive" terms were used. An example search phrase might be as follows: "triplenegative breast cancer anthracycline exposed" AND "biological marker" AND "disease-free survival".

\section{Data Extraction}

The following information were extracted from each eligible article: first author name, study location, publication date, number of participants, mean follow-up time, cutoff of ER/PR, TILs, p53 and Bcl-2.

\section{Results}

Altogether, 705 potentially relevant publications were found (Figure 1). After the removal of irrelevant titles (eg, biomarker gene analysis), neoadjuvant studies and abstracts comprising no evidence of survival data or isolated biomarker 


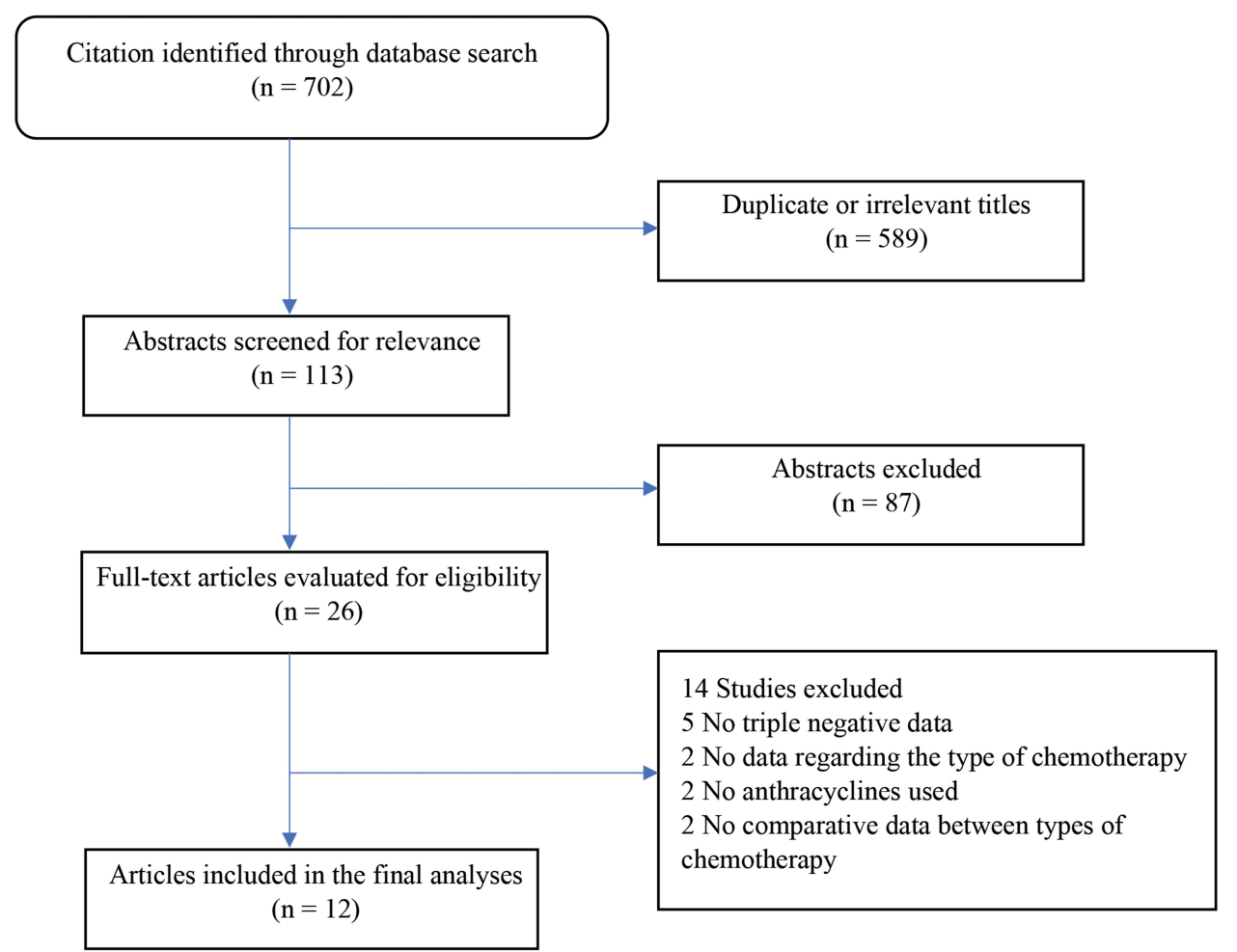

Figure I Flow diagram of the systematic literature review process.

analyses, 26 full-text articles remained eligible. Of those, we excluded studies that did not perform a subgroup analysis, those that did not mention the type of chemotherapy agent or did not contain anthracyclines and studies that did not show comparative data between the anthracycline and nonanthracycline subgroups. Twelve articles were included in the final analysis (Figure 1).

The markers of interest found in our analysis were tumour suppressor protein (p53), B cell lymphoma 2 antiapoptotic protein (Bcl-2) and TILs. The literature is contradictory on the predictive role of p53 expression, with two positive studies but with the opposite results and two negative studies totalling 2154 patients, 1466 of whom were exposed to adjuvant anthracyclines.

Encoded by the p53 gene, the p53 protein is known as a DNA damage checkpoint control inducing either DNA repair or apoptosis. ${ }^{11}$ Missense mutations or deletions lead to p53 nuclear-denatured protein synthesis, whose accumulation can be revealed by IHC. ${ }^{12}$ The markers' expression exceeds $60 \%$ in TNBC. $^{13}$

P53 overexpression (40\% of cases, cut-off 10\%) was associated with worse 10-year DFS $(p=0.005)$ and OS $(p=0.034)$ in a retrospective analysis $(n=135)$ in triplenegative patients treated with adjuvant anthracycline-based chemotherapy, the relative risk (RR) of relapse being five times higher in $\mathrm{p} 53$-positive cases $(\mathrm{RR}=5.4,95 \% \mathrm{CI}$ [1.420.8], $\mathrm{p}=0.013) .{ }^{14}$ On the contrary, a significantly better 10 year DFS rate $(57 \%)$ was found in p53-positive TNBC patients $(n=1088)$ who received anthracyclines $(p=0.036)$, but the difference was not significant in the subgroup exposed to non-anthracycline chemotherapy. ${ }^{15}$

Two studies showed no impact on survival for p53positive or -negative anthracycline-exposed TNBC patients, as no difference in 10-year DFS or OS was reported either in a large study $(n=678)^{14}$ or in a small study $(n=253)$; the latter study also assessed the predictive role of p53 in 44 triplenegative cases (hazard ratio $(\mathrm{HR})=0.68,95 \%$ CI $[0.22-$ 2.11]; $\mathrm{p}=0.55) .{ }^{16}$ Nevertheless, significantly better 10 -year DFS and OS $(p=0.006$ and $p=0.005$, respectively) were reported in combined score $\mathrm{p} 53$-TIL co-expression. ${ }^{17}$

Another relevant marker was Bcl-2, demonstrating a positive predictive role for anthracycline response in patients with low protein expression. Bcl-2 was identified more than 20 years ago in follicular lymphoma and is described in approximately $40-50 \%$ of non-metastatic triplenegative cases as a marker of favourable outcomes. ${ }^{18-20}$ Studies of cancer cell lines show that bcl2 might inhibit apoptosis induced by anthracyclines. ${ }^{21}$ These three analyses 
included 1279 patients of whom 675 were exposed to anthracyclines. All studies were positive, with the Asian analysis finding a correlation with survival only in the non-basal-like subtype rather than in the entire triple-negative cohort. ${ }^{22-24}$

Bcl-2 appeared to predict anthracycline response in a retrospective-prospective analysis $(n=600)$, showing better 5-year DFS in bcl2-negative cases than those treated with a CMF regimen (cyclophosphamide/methotrexate/ fluorouracil) or receiving no systemic treatment $(\mathrm{HR}=0.61,95 \%$ CI [0.44-0.84]; $\mathrm{p}=0.003)$. Surgical tissue specimens were reassessed by IHC and bcl2-negative was found in $70 \%$ of the cases. ${ }^{22}$

In another retrospective study wh examined immunohistochemical expression of Bcl-2 in 492 triple-negative breast cancers (TNBCs) using tissue microarrays, no significant association between Bcl-2 expression and clinical outcome was reported; however, the authors did find a three times higher HR for death in the non-basal-like cohort Bcl-2-positive subgroup (HR for five-year $\mathrm{OS}=3.04,95 \%$ CI [1.04-8.91], $\mathrm{p}=0.033)$. Strong predictive values for $\mathrm{DFS}(\mathrm{HR}=4.479$, 95\% CI [1.743-11.51], $\mathrm{p}=0.002)$ and $\mathrm{OS}(\mathrm{HR}=4.174,95 \%$ CI [1.617-10.775], $\mathrm{p}=0.003$ ) were demonstrated in a multivariate analysis. ${ }^{23}$

Bcl-2 expression was found to be significantly associated with survival in a population $(n=187)$ of which 164 received adjuvant anthracyclines: $\mathrm{RFS}(\mathrm{HR}=3.73,95 \% \mathrm{CI}$ [1.474-9.445], $\mathrm{p}=0.005)$, BCSS $(\mathrm{HR}=4.25,95 \%$ CI [1.372-13.174], $\mathrm{p}=0.012)$ and $\mathrm{OS}(\mathrm{HR}=4.32,95 \% \mathrm{CI}[1 .-$ 469-12.717], $\mathrm{p}=0.007){ }^{24}$

Four of five studies $(n=3134)$, comprising 1332 breast cancer patients treated by adjuvant anthracyclines, showed that the presence of tumor-infiltrating lymphocytes (TILs) and their composition show significant associations with prognosis and response to cancer treatments and can be an independent predictive marker.

Breast cancer was long considered to be a less immunogenic disease; however, recent preclinical studies have found that the chemotherapeutic agents, mainly anthracyclines, can cause immunogenic death by inviting and stimulating immune cells and finally inducing immunemediated cytotoxic cell death. ${ }^{25,26}$ TILs have also been associated with highly proliferative breast cancer subtypes and have been reported as a positive prognostic factor, especially in the triple-negative phenotype. ${ }^{27,28}$

TILs can be assessed in two ways: 1) using full-face H\&E sections (ie by counting all the mononuclear cells (lymphocytes and plasma cells) in the stromal compartment within the borders of the invasive tumour and reporting the percentage (TILs score)) and 2) immunohistochemical staining. ${ }^{29}$ Antibodies used in IHC are directed against the antigens of cytotoxic T-cells cluster of differentiation 8 (CD8), T-helper cells cluster of differentiation 4 (CD4), anti-T-regulatory lymphocytes forkhead box $\mathrm{p} 3$ (FOXp3) or the cluster of differentiation 3 (CD3) that detects both the CD8 and the CD4 T-cell population. The lymphocytic infiltrate is qualified as cytotoxic (CD8+), regulatory $(\mathrm{FOXp} 3+)$ or TILs non-specified $(\mathrm{CD} 3+) .^{30}$

High TIL expression assessed by CD3, CD8 and CD4 expression has been found to be correlated with a better five-year DFS rate $(86.7 \%)$ in cases who received anthracyclines compared with $35.1 \%$ for untreated patients (odds ratio=12, 95\% CI [2.34-61.55], $\mathrm{p}=0.0016$ ) in ER-negative breast cancer patients $(n=255)$. A better but not significantly different outcome was found for anthracyclinesexposed patients than those treated by the CMF regimen in the TIL-high subgroup (5-year DFS $86.7 \%$ vs $70.8 \%$, $\mathrm{p}=0.2346$ ). High CD3 expression established for a cut-off of six positive cells was associated with a reduced RR of $75 \% \quad$ (HR for $\mathrm{DFS}=0.25, \quad 95 \%$ CI [0.09-0.66], $\mathrm{p}=0.0056){ }^{31}$

In another study in which intratumoral T-cell infiltration was analysed using IHC CD8 and FOXp3 assessment infiltration, the presence of CD8 was associated with an increased benefit from adding epirubicin to $\mathrm{CMF}$ regiment compared with $\mathrm{CMF}$ regiment alone in the ER-negative population $(\mathrm{n}=1684$; HR for $\mathrm{BCSS}=0.60,95 \%$ CI $[0.37-$ $0.96], \mathrm{p}=0.039) .^{27}$

In three of the considered studies, TILs were assessed by H\&E as opposed to IHC. An entity called lymphocytepredominant breast cancer was defined as a lymphocyte infiltrate equal to or greater than $50 \%$. A categorical variable defined as an incremental $10 \%$ increase in the proportion of the mononuclear cells in contact with the tumour or within the stroma was also defined. In a post hoc analysis of 781 patients from two randomised Phase III trials, there was no survival difference in the anthracycline treated and no chemotherapy groups $(\mathrm{p}=0.55$ and $\mathrm{p}=0.44$ for intratumoral-TIL and stromal-TIL, respectively). ${ }^{32}$ For each $10 \%$ increase in stromal-TIL, a large retrospective analysis of 897 TNBC patients found a significant association with an anthracycline-containing regimen, with a $40 \%$ risk of death reduction $(\mathrm{HR}=0.60,95 \%$ CI $[0.48-0.76], \mathrm{p}=0.016) .{ }^{33}$ Finally, one of the largest studies evaluating the prognostic and predictive role of TILs in node-positive patients found-for each $10 \%$ increase in intratumoral and stromal lymphocytic infiltrations in ER-negative/HER2-negative patients $(n=256)$ 
treated with anthracyclines-a $70 \%$ RR reduction is $(\mathrm{HR}=0.30,95 \%$ CI $[0.11-0.81], \mathrm{p}=0.018) .{ }^{34}$

\section{Discussion}

This is the first systematic review of a biomarker's predictive role for anthracycline chemotherapy in triplenegative breast cancer. Other meta-analyses have examined the predictivity of the chemotherapy response of TILs in the neoadjuvant setting, showing the better pathological complete response for high lymphocytic infiltration; however, in the adjuvant setting, despite the good prognosis of high TILs, studies show that anthracyclines cannot be spared in this population. Indeed, two of the analyses show a significant benefit from adding epirubicin to CMF, namely, a $40 \%$ reduction in the risk of death due to breast cancer for every $10 \%$ increment in TILs. ${ }^{35,36}$ Regarding Bcl-2 expression, studies are in favour of sparing anthracyclines in Bcl-2-positive TNBC cases.
In this study, to perform a more comprehensive analysis than those in the literature, the triple-negative phenotype was extended to include the ER-negative and ER-negative/HER2negative subtypes; however, our systematic review only comprised 12 studies (Table 1). The restriction to the English language may have led us to miss important studies published in other languages. A total of 3473 anthracyclines treated patients were included, but the various chemotherapy regimens used to represent a confounding factor made it difficult to compare survival data across chemotherapy regimens. Further, the methods of protein detection and cut-offs varied between the studies, even for the same marker. However, we included studies that used two detection methods for TILs.

Regarding the $\mathrm{p} 53$ biomarker, four relevant studies (two positive) were included in our analysis; however, no conclusions could be drawn. The study conducted by Bae et al found a significant survival benefit for high p53 expression exposed to a doxorubicin cyclophosphamide regimen compared with other types of chemotherapy. ${ }^{15}$ An interesting

Table I Description of the Articles Included in the Final Analysis

\begin{tabular}{|c|c|c|c|c|c|c|}
\hline Study, Year & Marker & Method & $\begin{array}{l}\text { No. of } \\
\text { Patients }\end{array}$ & $\begin{array}{l}\text { Patient } \\
\text { Type }\end{array}$ & Chemotherapy & Outcome Measured \\
\hline Chae $2009^{14}$ & P53 & $\mathrm{IHC}$ & 135 & TNBC & A & RFS, RR=5.4 95\% Cl [1.4-20.8], $p=0.013$ \\
\hline Bae $2018^{15}$ & P53 & $\mathrm{IHC}$ & 1088 & TNBC & $\begin{array}{l}A C \text { vs } A C-D \text { vs } F A C \text { vs } \\
C M F\end{array}$ & 10-year DFS $p=0.036$ \\
\hline Lee $2018^{16}$ & P53 & $\mathrm{IHC}$ & 678 & TNBC & A & 10-year DFS \\
\hline Xu $2017^{17}$ & P53 & $\mathrm{IHC}$ & 253 & TNBC & A & DFS, HR=0.68, 95\% Cl [0.22-2.I I], p =0.55 \\
\hline $\begin{array}{l}\text { Abdel-Fatah } \\
2013^{22}\end{array}$ & $\mathrm{Bcl}-2$ & $\mathrm{IHC}$ & 600 & TNBC & A vs CMF & $\begin{array}{l}\text { Five-year DFS, HR=0.6I, 95\% Cl [0.44-0.84], } \\
p=0.003\end{array}$ \\
\hline Choi $2014^{23}$ & $\mathrm{Bcl}-2$ & $\mathrm{IHC}$ & 492 & $\begin{array}{l}\text { Non-basal- } \\
\text { like }\end{array}$ & A & $\begin{array}{l}D F S, H R=4.479,95 \% \mathrm{Cl}[1.743-|| .5 \mid] \\
P=0.002\end{array}$ \\
\hline $\begin{array}{l}\text { Bouchalova } \\
2015^{24}\end{array}$ & $\mathrm{Bcl}-2$ & $\mathrm{IHC}$ & 187 & TNBC & A & $\begin{array}{l}\text { RFS, HR=3.73, 95\% Cl [I.474-9.445], } \\
P=0.005\end{array}$ \\
\hline West $2015^{31}$ & TIL & $\mathrm{IHC}$ & 255 & ER- & A & $\begin{array}{l}\text { DFS, HR }=0.25,95 \% \mathrm{Cl}[0.09-0.66] \\
p=0.0056\end{array}$ \\
\hline Ali $2014^{27}$ & TIL & $\mathrm{IHC}$ & 1684 & ER- & A-CMF vs CMF & $\begin{array}{l}B C S S, H R=0.60,95 \% \mathrm{Cl}[0.37-0.96] \\
P=0.039\end{array}$ \\
\hline Dieci $2015^{32}$ & TIL & $\mathrm{H} \& \mathrm{E}$ & 781 & ER- & A vs no $\mathrm{CHT}$ & OS, $H R p=0.5$ \\
\hline Pruneri $2015^{33}$ & TIL & H\&E & 897 & TNBC & $A C+C M F$ vs $C M F$ & OS, $\mathrm{HR}=0.60,95 \% \mathrm{Cl}[0.48-0.76], \mathrm{p}=0.016$ \\
\hline Loi $2013^{34}$ & TIL & H\&E & 256 & ER- & A & RFS, $H R=0.30,95 \% \mathrm{Cl}[0.1 \mathrm{I}-0.8 \mathrm{I}], \mathrm{p}=0.018$ \\
\hline
\end{tabular}

Abbreviations: A, anthracycline-based chemotherapy; AC, doxorubicin cyclophosphamide; AC-D, doxorubicin cyclophosphamide followed by docetaxel; CMF, cyclophosphamide methotrexate 5-fluorouracil; DFS, disease-free survival; ER-, oestrogen receptor-negative; FAC, 5-fluorouracil doxorubicin cyclophosphamide; HR, hazard ratio; $\mathrm{H} \& \mathrm{E}$, haematoxylin and eosin; IHC, immunohistochemistry; OS, overall survival; RR, relative risk; RFS, relapse-free survival; TIL, tumor-infiltrating lymphocyte; TNBC, triplenegative breast cancer. 
finding was the association with TILs, which translated into better survival in anthracycline-exposed TNBC cases.

Our study does have some limitations. First, all included studies were retrospective cohort studies related to missing data about TIL measurement and Bcl-2. Next, the variation in the definition of high/low TIL level, and the timeline(s) used for PFS and OS among the included studies can affect the accuracy of the results.

\section{Conclusion}

The p53 overexpression seems to be a negative predictive factor, while TILs and Bcl-2 are positively and negatively correlated, respectively, with better survival in early-stage TNBC patients treated with anthracycline. These conclusions are based on retrospective and post hoc analyses. With extensive research, a meta-analysis might allow us to find predictive factors for anthracyclines in TNBC as well as other aggressive subtypes.

\section{Funding}

The authors did not receive a specific grant for this research from any funding agency in the public, commercial or not-for-profit sectors.

\section{Disclosure}

The authors report no conflicts of interest in this work.

\section{References}

1. Dawood S. Triple-negative breast cancer: epidemiology and management options. Drugs. 2010;70(17):2247-2258. doi:10.2165/11538150000000000-00000

2. Gamucci T, Vaccaro A, Ciancola F, et al. Recurrence risk in small, node negative, early breast cancer: a multicenter retrospective analysis. J Cancer Res Clin Oncol. 2013;139(5):853-860. doi:10.1007/s00432013-1388-2

3. Heys SD, Hutcheon AW, Sarkar TK, et al. Neoadjuvant docetaxel in breast cancer: 3-year survival results from the Aberdeen trial. Clin Breast Cancer. 2002;3(Suppl 2):S69-S74. doi:10.3816/CBC.2002.s.015

4. Azim HA Jr, de Azambuja E, Colozza M, Bines J, Piccart MJ. Longterm toxic effects of adjuvant chemotherapy in breast cancer. Ann Oncol. 2011;22(9):1939-1947. doi:10.1093/annonc/mdq683

5. Ghigo A, Li M, Hirsch E. New signal transduction paradigms in anthracycline-induced cardiotoxicity. Biochim Biophys Acta. 2016; pii: S0167-4889(16)30011-8.

6. Praga C, Bergh J, Bliss J, et al. Risk of acute myeloid leukemia and myelodysplastic syndrome in trials of adjuvant epirubicin for early breast cancer: correlation with doses of epirubicin and cyclophosphamide. J Clin Oncol. 2005;23:4179-4191. doi:10.1200/ JCO.2005.05.029

7. Tanner M, Isola J, Wiklund $\mathrm{T}$, et al. Topoisomerase II alpha gene amplification predicts favorable treatment response to tailored and dose-escalated anthracycline-based adjuvant chemotherapy in HER-2/ neu-amplified breast cancer: Scandinavian Breast Group Trial 9401. J Clin Oncol. 2006;24(16):2428-2436. doi:10.1200/JCO.2005.02.9264
8. Sinn HP, Schneeweiss A, Keller M, et al. Comparison of immunohistochemistry with PCR for assessment of ER, PR, and Ki-67 and prediction of pathological complete response in breast cancer. $B M C$ Cancer. 2017;17(1):124. doi:10.1186/s12885-017-3111-1

9. Hammond ME, Hayes DF, Dowsett M, et al. American Society of Clinical Oncology/College of American Pathologists guideline recommendations for immunohistochemical testing of estrogen and progesterone receptors in breast cancer. Arch Pathol Lab Med. 2010;134(6):907-922.

10. Wolff AC, Hammond ME, Schwartz JN, et al. American Society of Clinical Oncology/College of American Pathologists Guideline recommendations for human epidermal growth factor receptor 2 testing in breast cancer. $J$ Clin Oncol. 2007;25:118-145. doi:10.1200/JCO.2006.09.2775

11. Hamzehloie T, Mojarrad M, Hasanzadeh Nazarabadi M, Shekouhi S. The role of tumor protein 53 mutations in common human cancers and targeting the murine double minute 2-p53 interaction for cancer therapy. Iran J Med Sci. 2012;37(1):3-8.

12. Tsuda H, Hirohashi S. Association among p53 gene mutation, nuclear accumulation of the $\mathrm{p} 53$ protein and aggressive phenotypes in breast cancer. Int $J$ Cancer. 1994;57(4):498-503. doi:10.1002/ijc.291 0570410

13. Ilie SM, Bacinschi XE, Botnariuc I, Anghel RM. Potential clinically useful prognostic biomarkers in triple-negative breast cancer: preliminary results of a retrospective analysis. Breast Cancer. 2018;10:177-194.

14. Chae BJ, Bae JS, Lee A, et al. p53 as a specific prognostic factor in triple-negative breast cancer. Jpn J Clin Oncol. 2009;39(4):217-224. doi:10.1093/jjco/hyp007

15. Bae SY, Nam SJ, Jung Y, et al. Differences in prognosis and efficacy of chemotherapy by $\mathrm{p} 53$ expression in triple-negative breast cancer. Breast Cancer Res Treat. 2018;172(2):437-444. doi:10.1007/s10549018-4928-2

16. Lee M, Park IA, Heo SH, Kim YA, Gong G, Lee HJ. Association between $\mathrm{p} 53$ expression and amount of tumor-infiltrating lymphocytes in triple-negative breast cancer. J Pathol Transl Med. 2019;53 (3):180-187. doi:10.4132/jptm.2019.02.08

17. Xu YL, Yao R, Li J, et al. FOXC1 overexpression is a marker of poor response to anthracycline-based adjuvant chemotherapy in sporadic triple-negative breast cancer. Cancer Chemother Pharmacol. 2017;79 (6):1205-1213. doi:10.1007/s00280-017-3319-4

18. Gaulard P, d'Agay MF, Peuchmaur M, et al. Expression of the Bcl-2 gene product in follicular lymphoma. Am J Pathol. 1992;140 (5):1089-1095.

19. Zubair M, Hashmi SN, Afzal S, et al. Immunohistochemical expression of B cell lymphoma2 with clinicopathological correlation in triple negative breast cancers in northern Pakistan. Asian Pac J Cancer Prev. 2016;17(7):3619-3622.

20. Dawson SJ, Makretsov N, Blows FM, et al. BCL2 in breast cancer a favourable prognostic marker across molecular subtypes and independent of adjuvant therapy received [published correction appears in Br J Cancer. 2010 Sep 28;103(7):1137]. Br J Cancer. 2010;103 (5):668-675.

21. Ohmori T, Podack ER, Nishio K, et al. Apoptosis of lung cancer cells caused by some anti-cancer agents (MMC, CPT-11, ADM) is inhibited by Bcl-2. Biochem Biophys Res Commun. 1993;192(1):30-36. doi:10.1006/bbrc. 1993.1377

22. Abdel-Fatah TM, Perry C, Dickinson P, et al. Bcl-2 is an independent prognostic marker of triple negative breast cancer (TNBC) and predicts response to anthracycline combination (ATC) chemotherapy (CT) in adjuvant and neoadjuvant settings. Ann Oncol. 2013;24 (11):2801-2807. doi:10.1093/annonc/mdt277

23. Choi JE, Kang SH, Lee SJ, Bae YK. Prognostic significance of Bcl-2 expression in non-basal triple-negative breast cancer patients treated with anthracycline-based chemotherapy. Tumour Biol. 2014;35 (12):12255-12263. doi:10.1007/s13277-014-2534-4 
24. Bouchalova K, Svoboda M, Kharaishvili G, et al. BCL2 is an independent predictor of outcome in basal-like triple-negative breast cancers treated with adjuvant anthracycline-based chemotherapy. Tumour Biol. 2015;36(6):4243-4252. doi:10.1007/s13277-015-3061-7

25. Burugu S, Asleh-Aburaya K, Nielsen TO. Immune infiltrates in the breast cancer microenvironment: detection, characterization and clinical implication. Breast Cancer. 2016;2.

26. Casares N, Pequignot MO, Tesniere A, et al. Caspase-dependent immunogenicity of doxorubicin-induced tumor cell death. $J$ Exp Med. 2005;202(12):1691-1701. doi:10.1084/jem.20050915

27. Ali HR, Provenzano E, Dawson SJ, et al. Association between CD8+ T-cell infiltration and breast cancer survival in 12,439 patients. Ann Oncol. 2014;25(8):1536-1543. doi:10.1093/annonc/mdu191

28. Ibrahim EM, Al-Foheidi ME, Al-Mansour MM, Kazkaz GA. The prognostic value of tumor-infiltrating lymphocytes in triple-negative breast cancer: a meta-analysis. Breast Cancer Res Treat. 2014;148 (3): $467-476$.

29. Adams S, Gray RJ, Demaria S, et al. Prognostic value of tumor-infiltrating lymphocytes in triple-negative breast cancers from two phase III randomized adjuvant breast cancer trials: ECOG 2197 and ECOG 1199. J Clin Oncol. 2014;32:2959-2966. doi:10.1200/ JCO.2013.55.0491

30. Ladoire S, Mignot G, Dalban C, et al. FOXP3 expression in cancer cells and anthracyclines efficacy in patients with primary breast cancer treated with adjuvant chemotherapy in the phase III UNICANCER-PACS 01 trial. Ann Oncol. 2012;23(10):2552-2561. doi:10.1093/annonc/mds028

31. West NR, Milne K, Truong PT, Macpherson N, Nelson BH, Watson PH. Tumor-infiltrating lymphocytes predict response to anthracycline-based chemotherapy in estrogen receptor-negative breast cancer. Breast Cancer Res. 2011;13(6):R126. doi:10.1186/ bcr3072
32. Dieci MV, Mathieu MC, Guarneri V, et al. Prognostic and predictive value of tumor-infiltrating lymphocytes in two phase III randomized adjuvant breast cancer trials. Ann Oncol. 2015;26(8):1698-1704. doi:10.1093/annonc/mdv239

33. Pruneri G, Vingiani A, Bagnardi V, et al. Clinical validity of tumor-infiltrating lymphocytes analysis in patients with triple-negative breast cancer. Ann Oncol. 2016;27(2):249-256. doi:10.1093/annonc/mdv571

34. Loi S, Sirtaine N, Piette F, et al. Prognostic and predictive value of tumor-infiltrating lymphocytes in a phase III randomized adjuvant breast cancer trial in node-positive breast cancer comparing the addition of docetaxel to doxorubicin with doxorubicin-based chemotherapy: BIG 02-98. J Clin Oncol. 2013;31(7):860-867. doi:10.1200/JCO.2011.41.0902

35. Wang K, Xu J, Zhang T, Xue D. Tumor-infiltrating lymphocytes in breast cancer predict the response to chemotherapy and survival outcome: a meta-analysis. Oncotarget. 2016;7(28):44288-44298. doi:10.18632/oncotarget.9988

36. Carbognin L, Pilotto S, Nortilli R, et al. Predictive and prognostic role of tumor-infiltrating lymphocytes for early breast cancer according to disease subtypes: sensitivity analysis of randomized trials in adjuvant and neoadjuvant setting. Oncologist. 2016;21(3):283-291. doi:10.1634/theoncologist.2015-0307

\section{Publish your work in this journal}

Cancer Management and Research is an international, peer-reviewed open access journal focusing on cancer research and the optimal use of preventative and integrated treatment interventions to achieve improved outcomes, enhanced survival and quality of life for the cancer patient.
The manuscript management system is completely online and includes a very quick and fair peer-review system, which is all easy to use. Visit http://www.dovepress.com/testimonials.php to read real quotes from published authors. 